\title{
Pressure Drop Evolution of a Medium-Performance Fibrous Filter during Loading of Mist Aerosol Particles
}

\author{
Surajit Tekasakul ${ }^{1 *}$, Punnida Suwanwong ${ }^{1}$, Yoshio Otani ${ }^{2}$, Perapong Tekasakul ${ }^{3}$ \\ ${ }^{1}$ Department of Chemistry, Faculty of Science, Prince of Songkla University, \\ Hat Yai, Songkhla 90112, Thailand \\ ${ }^{2}$ Department of Chemical Engineering, Graduate School of Natural Science and Technology, \\ Kanazawa University, Kakuma, Kanazawa 920-1192, Japan \\ ${ }^{3}$ Department of Mechanical Engineering, Faculty of Engineering, Prince of Songkla University, \\ Hat Yai, Songkhla 90112, Thailand
}

\begin{abstract}
Collection characteristics of mist aerosol particles by a medium-performance fibrous filter was studied by analyzing the effect of filtration velocity, numbers of filter, types of liquid with different physical properties (palm oil and propylene glycol) during the initial filtration period and the saturation behavior. The results show that the pressure drop evolutions during clogging increases when the filtration velocity increases because at high filtration velocity, the aerosol particles deposit on the collecting surface rapidly. Furthermore, the change in pressure drop increases with increasing numbers of filter. Particle size, concentration, viscosity and surface tension of the liquids have an influence on clogging. The deposition of palm oil aerosol particles is only made up of droplets deposited around the fibers while in the case of propylene glycol the droplets deposited around the fiber join to form bridges and liquid films on the surface of the filter so that the fibrous filter that is exposed to propylene glycol aerosol has a higher pressure drop. The result of saturation behavior study shows that the saturation pressure drop from the lower initial velocity is greater than that from the higher initial velocity. The retained mass on the filter sheet saturated with palm oil after the pressure drop is constant is higher than in the case of propylene glycol. Palm oil is more viscous than propylene glycol so that palm oil cannot re-entrain easily.
\end{abstract}

Keywords: Mist; Fibrous filter; Pressure drop; Size distribution.

* Corresponding author. Tel: +66-74-288-434; Fax:

$+66-74-212-918$

E-mail address: surajit.t@psu.ac.th

\section{INTRODUCTION}

Mist aerosol particles can be generated in several industrial production processes 
including chemical production, mechanical atomization, evaporation-condensation, entrainment by the gas flow in liquid-gas contactors, and other processes (Frising et al., 2005). People exposed to fine aerosol particles can have their skin contaminated and inhale them to cause damage to their lungs. Therefore for safety and health reasons it is necessary to remove these mist aerosol particles. Filtration is the most common method for removing aerosols as it is simple, versatile and economical (Hinds, 1999). At present, fibrous filtration is the most common means used to separate liquid aerosol particles from both industrial and household gas streams.

Fibrous filters are economically the most interesting filtration technology since they are both effective and simple to use. Some attempts have been conducted to investigate the behavior of various filter materials used to remove liquid aerosols. In general, during use the collection efficiency of the filter declines while the pressure drop increases (Letts et al., 2003). The behavior of fibrous filters with liquid particle loading is largely different from that with solid particle loading. When loaded with solid aerosols, dendrites are formed, and when loaded with liquid aerosols, bridges are formed (Walsh et al., 1996).

The effects of the time of filtration were studied by Walsh et al. (1996) and Contal et al. (2004). At the beginning of the filtration of liquid aerosols, the pressure drop and the penetration increased because the droplets were deposited around the fibers and the beads then became bigger and joined together to form bridges at the intersections of the fibers. This then caused a reduction of the collection surface of the filters. Subsequently all interstices were bridged and these bridges combined to form a liquid film on the surface of the filter. This effect generated the increase of pressure drop and the decrease of penetration. At the end of this clogging, an equilibrium was reached between the clogging, draining and re-entrainment phenomena. The pressure drop and penetration no longer changed because liquid film was formed throughout the thickness of the filter.

The influences of velocity were investigated by Contal et al. (2004) and Frising et al. (2005). As might be expected, at high filtration velocity, the liquid aerosol particles were rapidly deposited on the collecting surface causing an increase of the pressure drop. Initially, the penetration increased identically for each increase of filtration velocity until it reached a maximum value. Then the penetration decreased due to rearrangement of the liquid in the filter such as the forming of liquid bridges between fibers and at the fiber intersections. Furthermore, Contal et al. (2004) investigated the effects of filter properties and the physicochemical characteristics of the liquid on the change in pressure drop. During clogging by di-octyl phthalate (DOP) aerosol, the Whatman-type glass fiber medium had the highest change in the pressure drop because this filter was composed of fine fibers. The influence of the surface tension of aerosols generated from three liquids: decamethylcyclopentasiloxane (DMP), di-octyl phthalate (DOP) and glycerol, 
was examined and it was found that the filter that was exposed to the glycerol aerosol had the highest pressure drop. This is because glycerol has a higher surface tension (92 $\mathrm{mN} / \mathrm{m})$ than DOP $(35 \mathrm{mN} / \mathrm{m})$ and DMP (19 $\mathrm{mN} / \mathrm{m})$.

Although there have been some investigations of the collection characteristics of liquid aerosol by fibrous filters, this knowledge is limited. The aims of this research work are to investigate the collection characteristics of mist aerosol particles by fibrous filter using various basic operating parameters such as filtration velocity, numbers of filter and any relationships with the physicochemical properties of the liquids and to investigate the saturation characteristics of a medium-performance fibrous filter.

\section{Pressure Drop Modeling}

The pressure drop in a fibrous filter is the resistance to airflow across it. A consideration of the pressure drop across the filter medium is essential in choosing the best filter type for a particular application. The pressure drop is easily measured and can be used as a check on the flow fields on which collection mechanisms are based. More importantly, the measurement of the pressure drop across the filter medium plays a central role in the practical estimation of the collection efficiency. Ideally, filters that exhibit a high collection efficiency and a low pressure drop are most desirable.

Davies (1952) characterized the pressure drop across a dry fibrous filter, $\Delta p_{0}$, as

$$
\Delta p_{0}=\frac{u \mu_{g} Z}{d_{f}^{2}}\left[64 \alpha_{f}^{1.5}\left(1+56 \alpha_{f}^{3}\right)\right]
$$

where $u$ is the face velocity of the gas, $\mu_{g}$ is the gas viscosity, $Z$ is the filter thickness, $\alpha_{f}$ is the filter solidity or the filter packing density, and $d_{f}$ is the fiber diameter. This shows that the pressure drop is directly proportional to $Z$ and inversely proportional to $d_{f}^{2}$. It is also directly proportional to the gas velocity at the filter face, as expected for laminar flow inside the filter.

Even though the pressure drop has been extensively studied experimentally, very few modeling studies have been published to date that investigated the change in the pressure drop across a fibrous filter during a filtration.

Davies (1973) presented a modification of his own pressure drop model

$$
\Delta p=u \mu Z \frac{64 \alpha_{f}^{1.5}\left(1+56 \alpha_{f}^{3}\right)}{d_{f \text { Davies wet }}^{2}}
$$

where $\mu$ is the liquid viscosity, and the wet fiber diameter $d_{f \text { Davieswet }}$ is defined as

$$
d_{f \text { Davieswet }}=d_{f \text { Davies }} \sqrt{1+\frac{m_{l}}{\Omega \rho_{l} Z \alpha_{f}}}
$$

Here $m_{l}$ is the collected liquid mass, $\Omega$ is filtration surface area, $\rho_{l}$ is liquid density, and $d_{f \text { Davies }}$ can be calculated from (Penicot et al., 1999)

$$
d_{f \text { Davies }}=2 \sqrt{\frac{16 \mu Z \alpha_{f}^{3 / 2}\left(1+56 \alpha_{f}^{3}\right)}{K}}
$$


where $K$ is the slope of the straight line between $\Delta p$ and $u$.

Frising et al. (2005) showed that the pressure drop evolution occurred in four filtration stages. In the first stage, the liquid is only deposited on the surface of a fiber. No drainage or migration of liquid from one layer to the next is observed. The pressure drop is calculated by an adapted version of Davies' equation (1973):

$$
\begin{gathered}
\Delta p=64 \mu u d Z \frac{\left(\alpha_{f}+\alpha_{l}\right)\left(\alpha_{f}+\alpha_{l}\right)^{0.5}}{d^{2}{ }_{f \text { Davies wet }}} \\
{\left[1+16\left(\alpha_{f}+\alpha_{l}\right)^{2.5}\right]}
\end{gathered}
$$

where $\alpha_{l}$ is liquid packing density, and $d Z$ is the filter layer thickness which can be written as

$$
d Z=\frac{Z}{n p}
$$

Here $n p$ is the number of layers.

In the second stage, the liquid bridges between fibers and liquid films at the intersections of fibers are formed. Some of the collected liquid particles migrate to the next layer downstream. In this stage, the collection surface decreases due to the formation of liquid bridges and films. The flow will be largely disturbed by the liquid bridges and films. So the pressure drop expression has to take the modified filtration velocity into account:

$$
\begin{aligned}
\Delta p= & 64 \mu d Z \frac{\left(\alpha_{f}+\alpha_{\text {tube }}\right)\left(\alpha_{f}+\alpha_{l}\right)^{0.5}}{d^{2}{ }_{f \text { Davies wet }}} \\
& {\left[1+16\left(\alpha_{f}+\alpha_{l}\right)^{2.5}\right] \frac{u}{1-\alpha_{l}+\alpha_{\text {tube }}} }
\end{aligned}
$$

where $\alpha_{\text {tube }}$ is the liquid packing density at the end of the first stage.

In the third stage, all the collected liquid particles are supposed to migrate to the downstream layer due to capillary (wetting) or flow (migration) forces. The pressure drop is constant in this stage:

$$
\begin{aligned}
\Delta p= & 64 \mu d Z \frac{\left(\alpha_{f}+\alpha_{\text {tube }}\right)\left(\alpha_{f}+\alpha_{\text {film }}\right)}{d^{2}{ }_{f \text { Davies wet }}^{0.5}} \\
& {\left[1+16\left(\alpha_{f}+\alpha_{\text {film }}\right)^{2.5}\right] \frac{u}{1-\alpha_{\text {film }}+\alpha_{\text {tube }}} }
\end{aligned}
$$

where $\alpha_{\text {film }}$ is the maximum liquid packing density.

The fourth stage, is when all the layers are saturated by the collected liquid. All layers have a liquid packing density of $\alpha_{\text {film }}$, the collected liquid drains out of the filter.

This research focuses on studying the collection characteristics and performance of a fibrous filter as it collects mist aerosol particles. The evolution of the pressure drop of a fibrous filter was investigated only in the first filtration stage because at the beginning of filtration the deposit is made up of droplets deposited around the fibers and no drainage or migration of liquid from one layer to the next is observed. 


\section{EXPERIMENTAL SETUP}

The mist aerosols used in this study were generated from the Laskin nozzle. The size distribution and concentration of generated aerosols were determined to study their characteristics and stability. The liquids used for aerosol generation were propylene glycol and palm oil because their physical properties are different. An 8-stage Andersen sampler (Dylec, AN 200) with the cut sizes of 11.0, 7.0, $4.7,3.3,2.1,1.1,0.65$ and 0.43 micron was used to determine the aerosol concentration and the size distribution of the generated aerosol.

Performance of a single virgin filter was evaluated by determining the filtration efficiency (or penetration) and pressure drop using the propylene glycol liquid aerosol. The test filter is a medium-performance glass fiber filter. The thickness, the fiber diameter and the packing density are $0.56 \mathrm{~mm}, 3.65 \mu \mathrm{m}$ and 0.061 , respectively. The test filter was first weighed and then placed in the filter holder and the pressure drop was measured at different filtration velocities. Filter loading was monitored by measuring the pressure drop. After $30 \mathrm{~min}$, particle concentrations of the liquid aerosol were sampled upstream and downstream of the filter simultaneously using a laser particle counter (Royco, Portable 330B). The filtration velocities used were 5,7 , 10, 14, 21 and $28 \mathrm{~cm} / \mathrm{s}$.

To evaluate the collection characteristics of the mist aerosol particles by the fibrous filter, the relationship between pressure drop and collected mass at various conditions including numbers of filter sheets, filtration velocity, and physical properties of liquids were investigated. A schematic diagram of the experimental set-up for studying mist aerosol collection characteristics of a fibrous filter is shown in Fig. 1. Compressed air at a pressure of 1.4 bar was used to generate the mist aerosol. The flow rate of the compressed air introduced through the Laskin nozzle was 3.0 $\mathrm{L} / \mathrm{min}$. Only $1.0 \mathrm{~L} / \mathrm{min}$ of generated aerosol was introduced to the system and diluted with the clean air. Excessive liquid aerosol was removed by passing through a buffer chamber using a vacuum pump. The glass fiber filters were treated in the desiccator using silica gel at room temperature $\left(25^{\circ} \mathrm{C}\right)$ and $50 \%$ relative humidity for at least $24 \mathrm{~h}$. They were then weighed using a five-digit readability analytical balance (Sartorius, CP225D). The change in the pressure drop across a single filter and five filter sheets were investigated at four different filtration velocities: 7, 14, 21 and $28 \mathrm{~cm} / \mathrm{s}$, by adjusting the flow rate using a rotameter.

A single-filter was first weighed and then placed in the filter holder. Then the pressure drop was measured at different filtration velocities. Filter loading was monitored by measuring the pressure drop. The weight of the collected liquid particles on the filter was measured every 10 min until the pressure drop reached three times that of the initial value.

To highlight experimentally any possible redistribution of the liquid particles within the medium, five-filter experiments were 


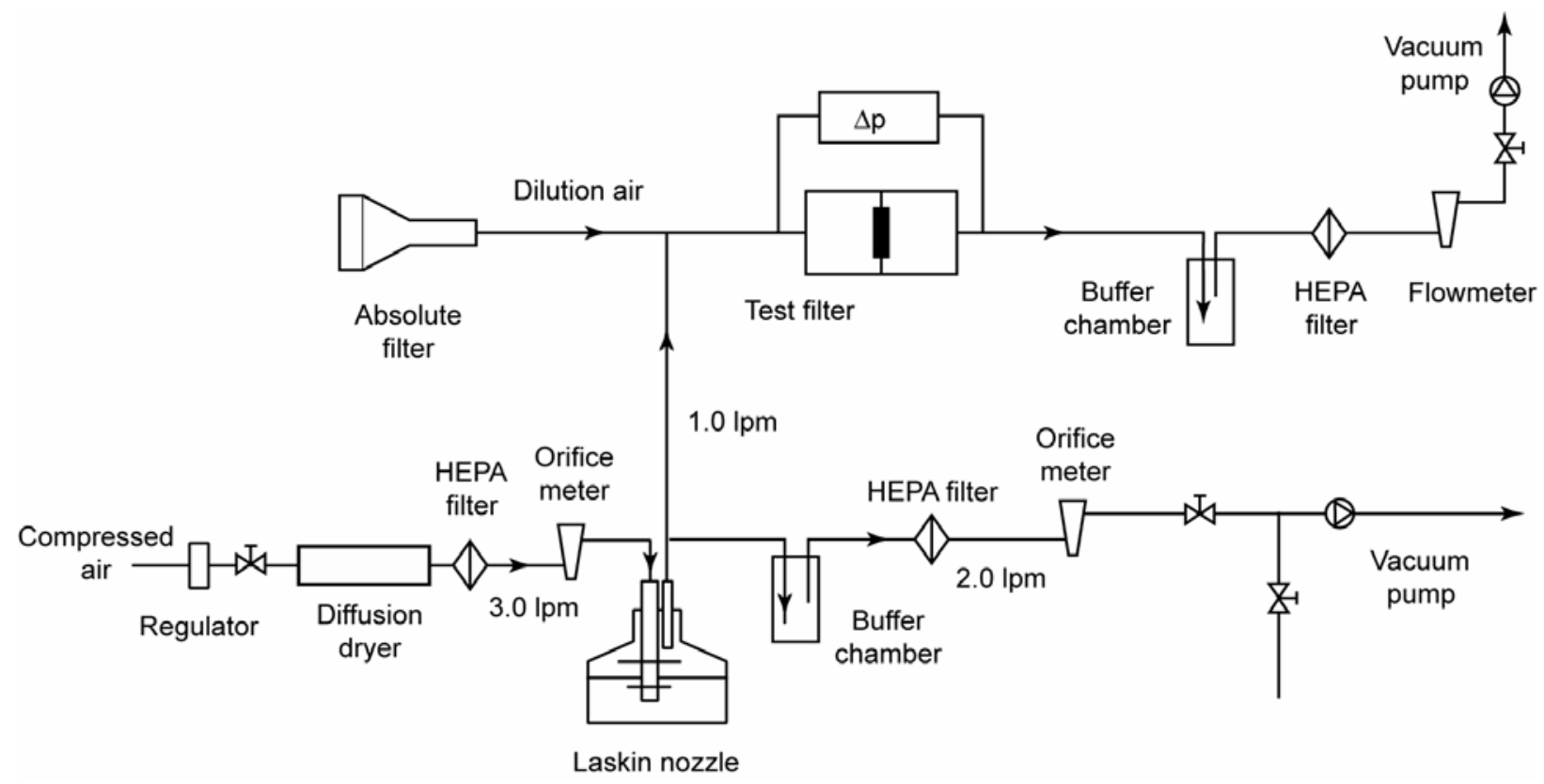

Fig. 1. The schematic representation of the experimental set-up for studying the collection characteristics of mist aerosol particles.

conducted. Each filter was first weighed and then placed in the filter holder in a series side by side. The weight of the collected liquid particles on each filter was measured every 10 min until the pressure drop reached three times that of the initial value.

The influence of the physical properties of the aerosol particles was investigated by using liquids with different physical properties. Propylene glycol and palm oil were chosen. The pressure drop across the single filter and five filters were measured at a filtration velocity of $14 \mathrm{~cm} / \mathrm{s}$.

The saturation characteristics of a mediumperformance fibrous filter (glass fiber filter) exposed to liquids (water, propylene glycol and palm oil) were studied by measuring the pressure drop. The filter was first weighed and immersed in a liquid (water, propylene glycol or palm oil) until it was saturated and then placed in the filter holder. Clean air passing through an absolute filter was introduced to the filter until the pressure drop was constant. The pressure drop was measured by a U-tube manometer. The retained liquid particles on a glass fiber filter were weighed using the same balance. Then the filtration velocity was reduced from the initial velocity to the minimum velocity of $1 \mathrm{~cm} / \mathrm{s}$. The constant pressure drop was recorded at each step of the reduced velocity.

\section{RESULTS AND DISCUSSION}

The characteristics of the aerosol to be used were first studied. Size distributions of propylene glycol and palm oil aerosol are shown in log-probability form in Figs. 2 (a) and (b), respectively. The distributions show lognormal behavior for both aerosol particles. 

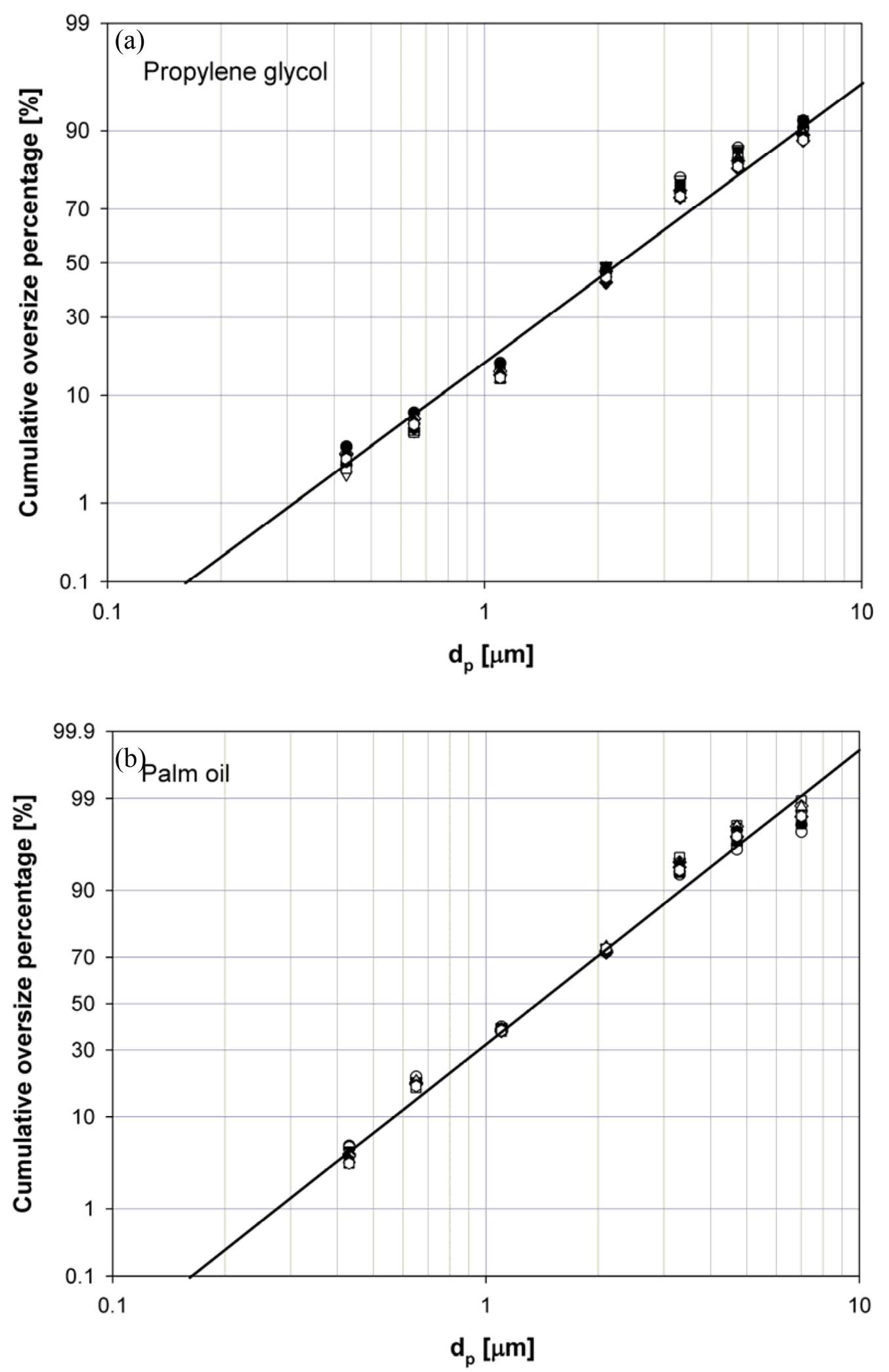

Fig. 2. Size distribution of oil mist generated from the Laskin nozzle shown in Log-probability plots for (a) propylene glycol aerosol, and (b) palm oil aerosol. 
Average mass median aerodynamic diameter (MMAD) and geometric standard deviation (GSD) of propylene glycol aerosol are about $2.30 \mu \mathrm{m}$ and $2.37 \mu \mathrm{m}$, respectively, while average MMAD and GSD of palm oil aerosol are $1.42 \mu \mathrm{m}$ and $2.10 \mu \mathrm{m}$, respectively. Results show a larger variation of concentration and collected mass of the propylene glycol aerosol than the palm oil aerosol, as seen in Fig. 3. The average aerosol concentrations of propylene glycol are 0.523 $\mathrm{g} / \mathrm{m}^{3}$ while the average aerosol concentrations of palm oil are $0.303 \mathrm{~g} / \mathrm{m}^{3}$. For identical operating conditions, the average concentration of propylene glycol is higher than that of palm oil because propylene glycol is less viscous than palm oil and is easier to be atomized by the Laskin nozzle.

Effects of particle size on the collection efficiency of a virgin filter using propylene glycol aerosol particles are shown in Fig. 4. The results indicate that the efficiency of a virgin filter slightly increases when the particle size increases for all filtration velocities. Moreover, the efficiency is nearly independent of particle sizes for filtration velocity of $5-21 \mathrm{~cm} / \mathrm{s}$. At the highest velocity of $28 \mathrm{~cm} / \mathrm{s}$, the difference in efficiency is significant for all particle sizes. However, at the same filtration velocity $(28 \mathrm{~cm} / \mathrm{s})$ the filter shows a better collection efficiency for larger particles than smaller particles. The generated propylene glycol aerosol has a rather large particle size at MMAD of $2.30 \mu \mathrm{m}$. Then the impaction mechanism plays an important role in collection of particles at this high velocity. The importance of the inertial impaction increases with increasing particle size and increasing flow velocity. The collection efficiencies are about $97 \%, 89 \%, 76 \%$ and $26 \%$ for the particle size of $1.0,0.7,0.5$ and $0.3 \mu \mathrm{m}$, respectively, at a filtration velocity of $28 \mathrm{~cm} / \mathrm{s}$. In addition, at the same particle size, particularly for small particles, the collection efficiency was higher at a lower filtration velocity. Brownian motion of small particles is believed to play an important role in this regime. Influence of Brownian motion is increased when particle size and flow velocity are decreased (Hinds, 1999).

The pressure drop across a filter is a parameter used to determine the collection performance in this study. The pressure drop is the resistance to the air flowing through the fibrous filter. The liquid used for mist generation in this experiment was propylene glycol. The pressure drop across the virgin filter after filtration for $30 \mathrm{~min}$ at different velocities is shown in Fig. 5. The results show that the pressure drop of the fibrous filter increases with increasing filtration velocity. This is commonly known however the increase is not linear.

Different clogging tests were conducted by varying the operating conditions, including filtration velocity, number of filter sheets and aerosol particles with different physical properties. The influence of each parameter is studied by eliminating the effect of other factors.

Fig. 6 represents the pressure drop evolutions of a single filter as a function of collected mass during clogging by propylene glycol aerosol with different filtration 


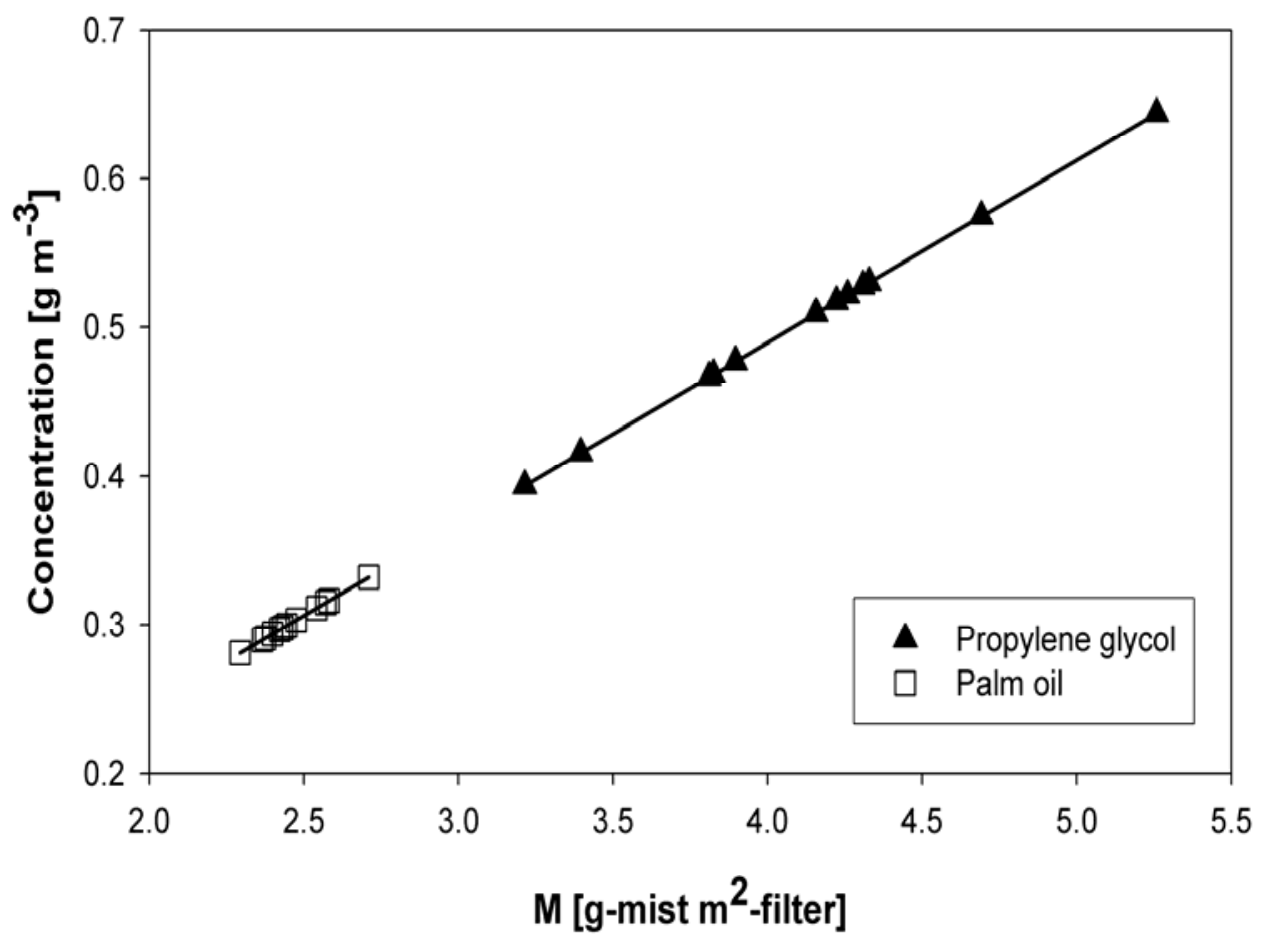

Fig. 3. Concentration of oil mist generated from the Laskin nozzle as a function of the collected mass.

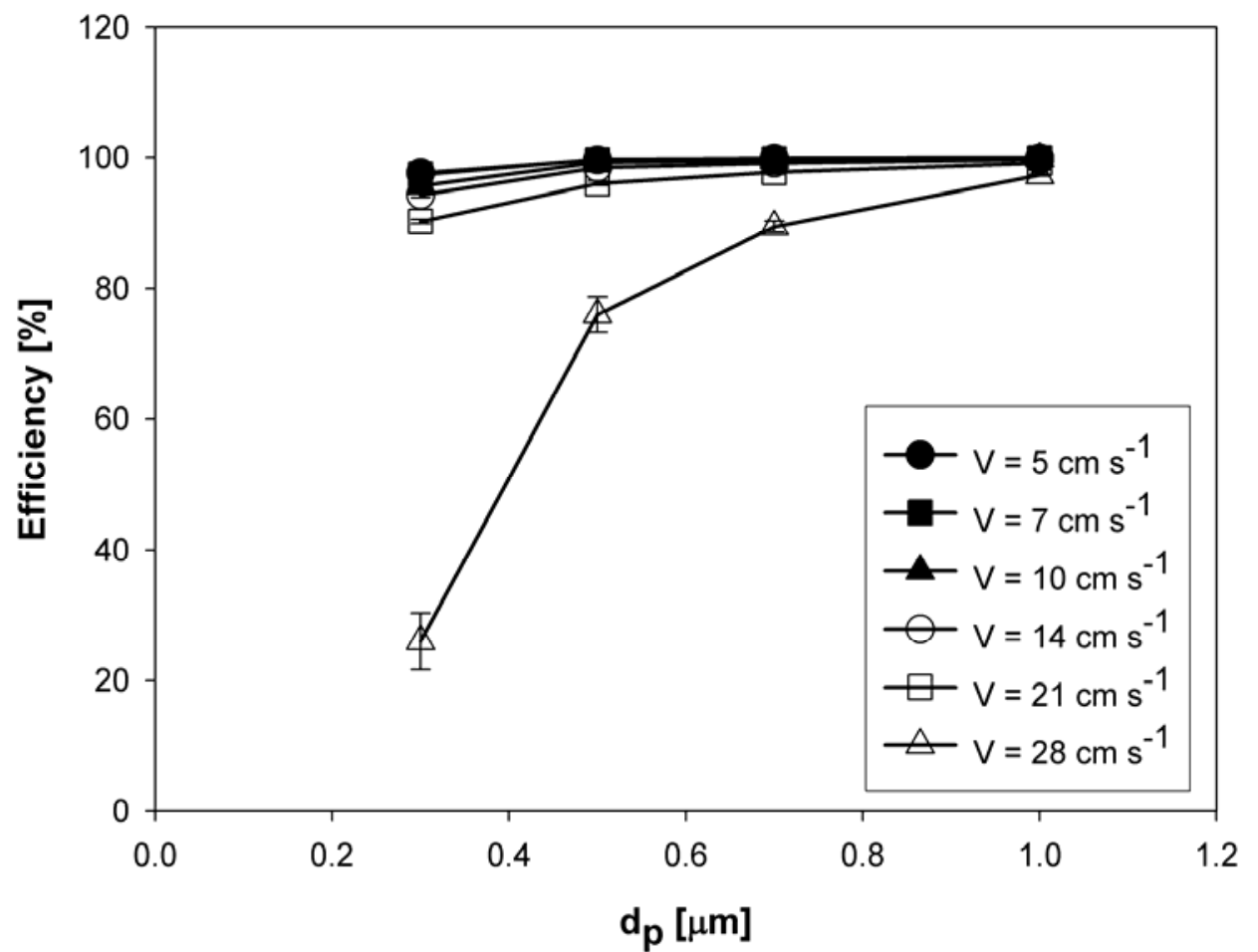

Fig. 4. Effect of particle size on the efficiency of a filter using propylene glycol particles after filtration for $30 \mathrm{~min}$ at different velocities. 


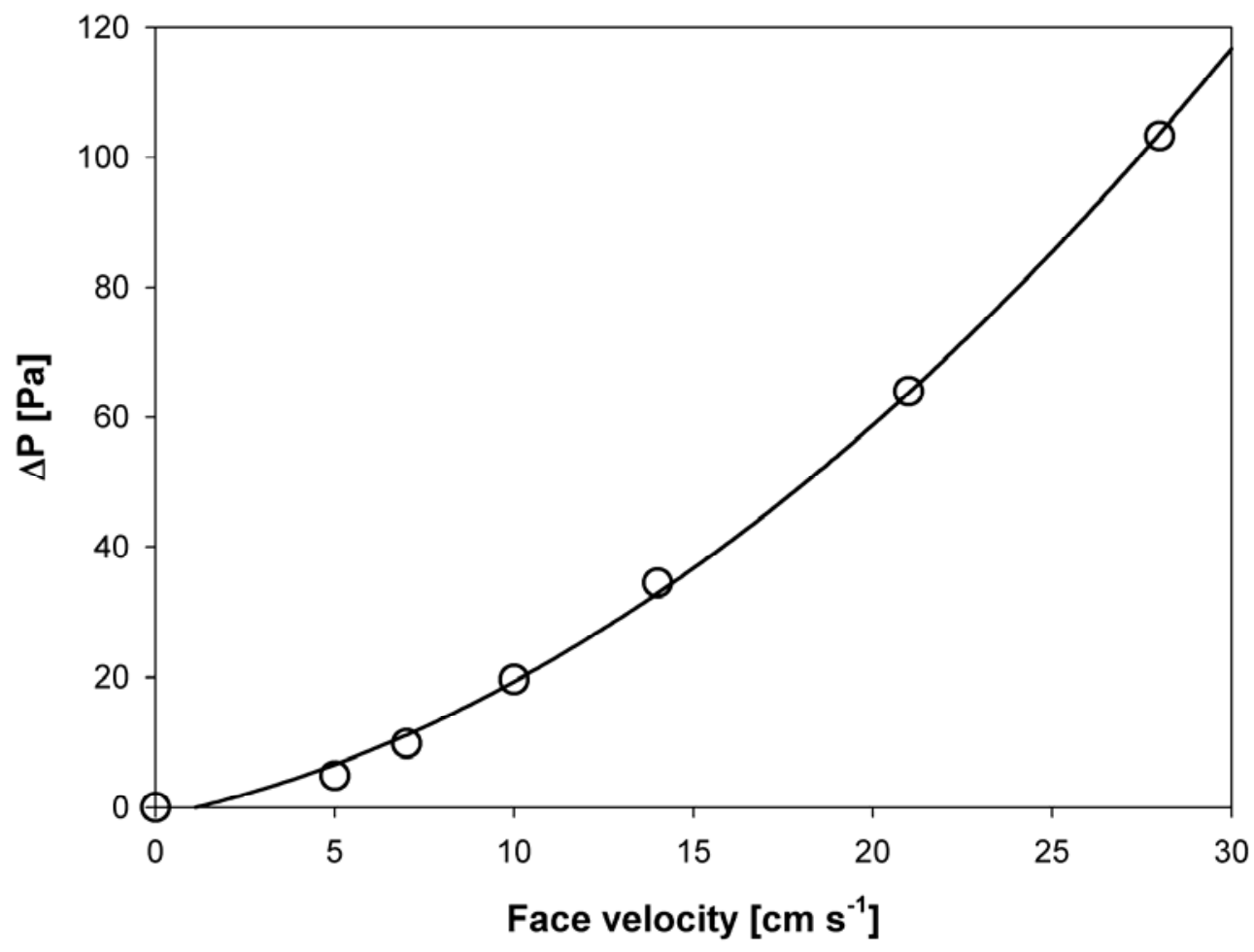

Fig. 5. Pressure drop across a filter where the oil mist is propylene glycol and after filtration for 30 min at different velocities.

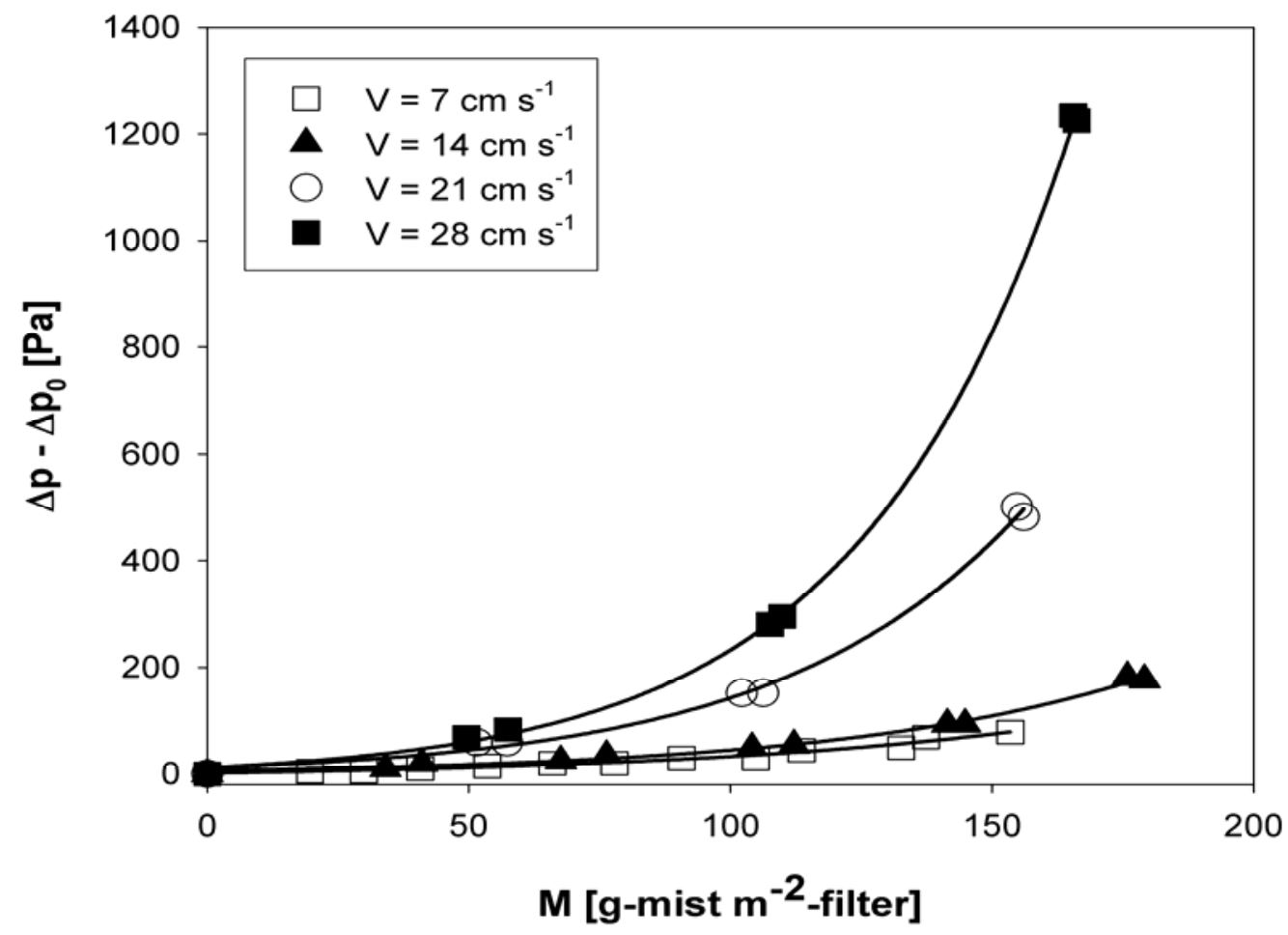

Fig. 6. Pressure drop evolutions of a single filter as a function of collected mass during clogging by propylene glycol aerosol with different filtration velocities. 
velocities: 7, 14, 21 and $28 \mathrm{~cm} / \mathrm{s}$. For the same filtration time, the mass collected (M) on the filter at high filtration velocity is greater than that at low filtration velocity since the liquid aerosol particles deposit rapidly on the collecting surface. This effect causes the pressure drop of a single filter to increase as the collected mass increases, which is similar to the result of Contal et al. (2004).

The pressure drop evolutions for 1 and 5 filters at filtration velocities of 14 and $28 \mathrm{~cm} / \mathrm{s}$ as a function of collected mass are shown in Figs. 7 (a) and (b), respectively. The generated aerosol was propylene glycol. Results show that the change in pressure drop increases with the increasing number of filter sheets for all velocities. In the initial period, the pressure drop of five filters is five times higher than expected. From Fig. 7 (b), after some time the pressure drop of a single filter intersected with that of five filters. This is because at a higher filtration velocity, the filter was rapidly saturated with liquid aerosol particles and these liquid aerosol particles were mostly collected on the first filter sheet so that the first filter sheet has a dominant effect on the pressure drop evolutions over all other filter sheets. For five filters, while the liquid aerosol particles were collected on the surface of the filter, these aerosol particles can partially penetrate through the thickness of the medium. When the number of filter sheets increases, the change in the pressure drop increases since the pressure drop is directly proportional to the thickness of the filter.

Comparison of the pressure drop across a single filter for different aerosols is shown in
Fig. 8 when the glass fiber filters are exposed to liquid aerosol particles of propylene glycol and palm oil at a filtration velocity of $14 \mathrm{~cm} / \mathrm{s}$. It was found that, the pressure drop curves can be divided into two periods. In the initial period, the pressure drop is linearly related to the collected mass. The clogging of the filter by the palm oil aerosol is higher than that by the propylene glycol aerosol. This is because of the particle size effect. Palm oil mist has a smaller particle size and a larger surface area than that of the propylene glycol. Thus the amount of collected palm oil aerosol on the surface of the filter medium is greater than for the propylene glycol aerosol as shown in Figs. 9 (a) and (b). After some time, the pressure drop increases at a higher rate. The fibrous filter that is exposed to the propylene glycol aerosol has a higher pressure drop. This is because propylene glycol is less viscous (64.6 cP) than palm oil (94.6 cP) and therefore easier to be atomized by the Laskin nozzle, so the concentration of propylene glycol is higher than that of palm oil and the large propylene glycol droplets deposited on the fibrous filter join to form bridges at the intersection of fibers. Subsequently all interstices are bridged and these bridges combine to form a liquid film on the surface of the filter medium as shown in Figs. 10 (a) and (b). However, since palm oil is not only more viscous but also has a slightly less surface tension $(42.2 \mathrm{mN} / \mathrm{m})$ than propylene glycol $(47.6 \mathrm{mN} / \mathrm{m})$, the palm oil aerosol deposit is only made up of droplets collected around the fibers [Fig. 10 (b)].

Fig. 11 represents the comparison between the experimental values of the pressure drop 

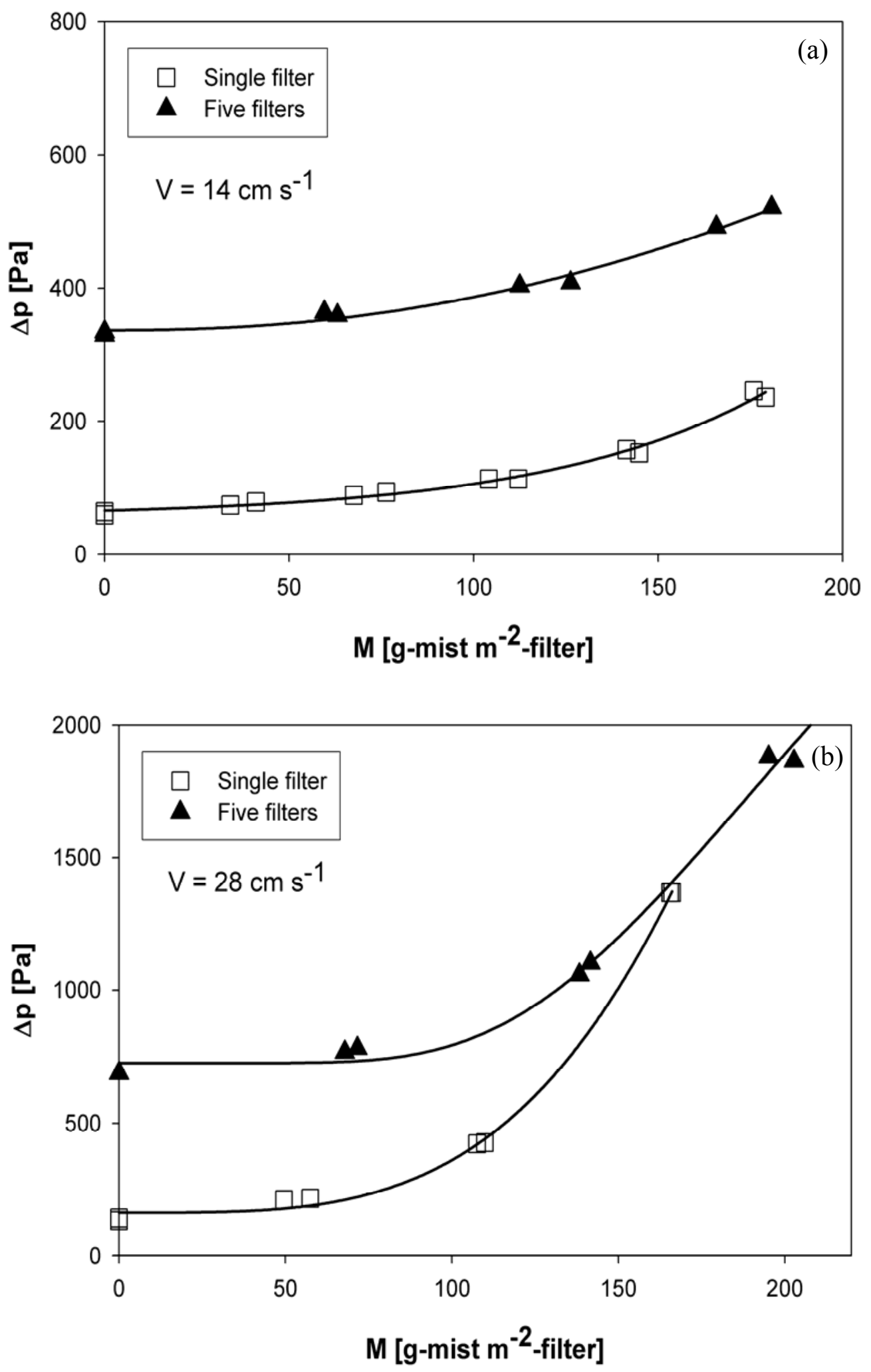

Figs. 7. Effect of the number of filter sheets on the pressure drop evolutions as a function of collected mass at filtration velocities of (a) $14 \mathrm{~cm} / \mathrm{s}$ and (b) $28 \mathrm{~cm} / \mathrm{s}$. 


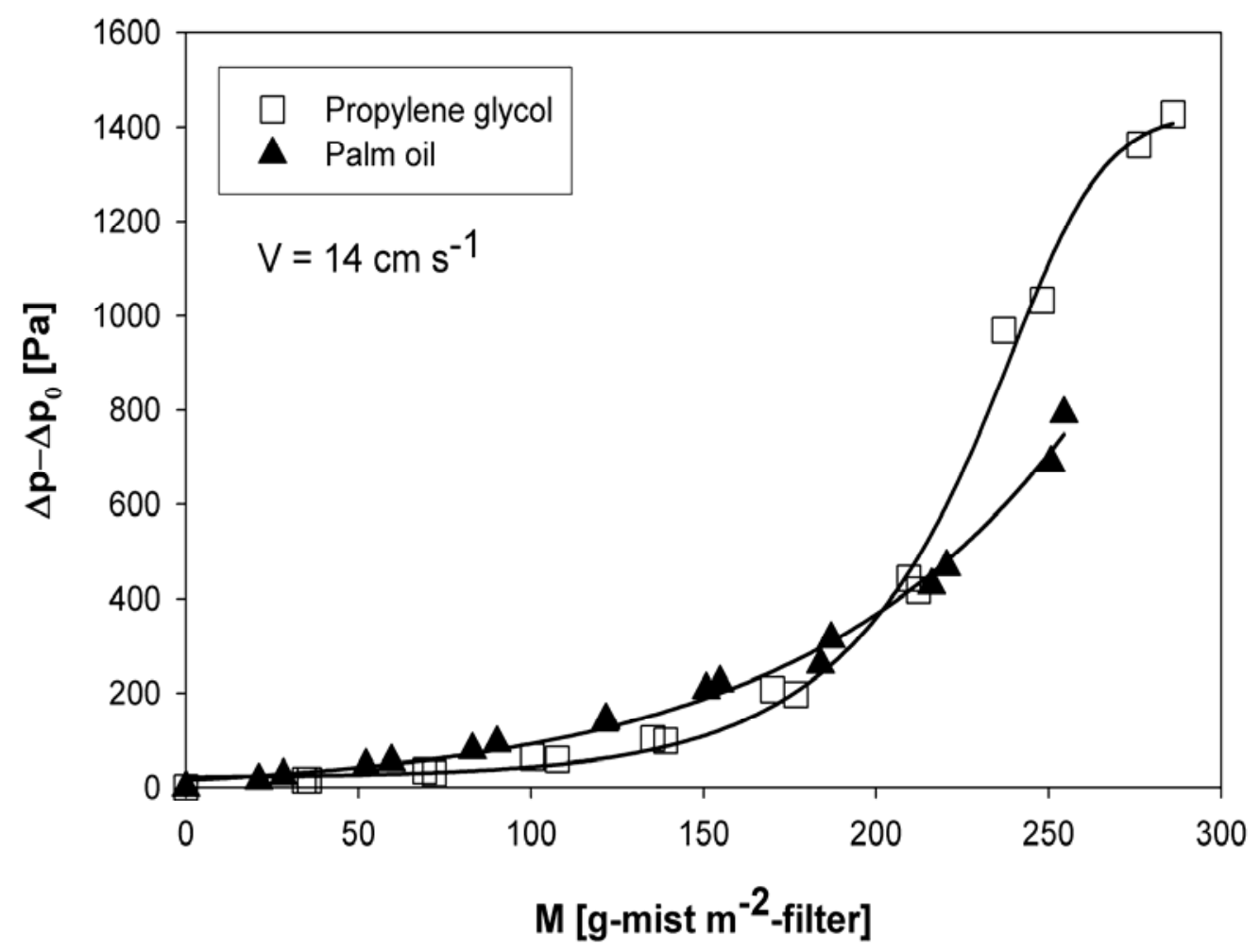

Fig. 8. The change in pressure drop across a single filter as a function of collected mass at $14 \mathrm{~cm} / \mathrm{s}$.
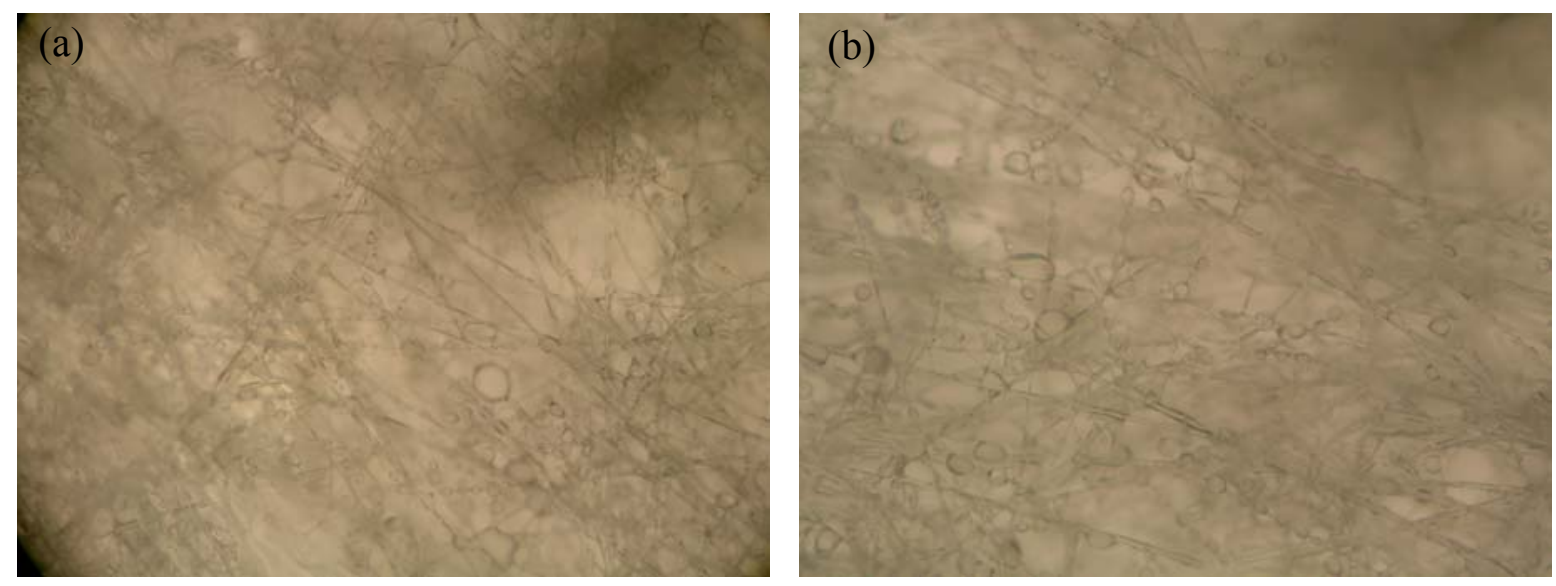

Fig. 9. Optical microscope photographs of a single filter exposed to (a) propylene glycol and (b) palm oil aerosol for $20 \mathrm{~min}(100 \times)$.

and those calculated from the Davies' equation (5) for the pressure drop evolutions of a single filter as a function of the collected mass during clogging by the propylene glycol aerosol with different filtration velocities of 7 ,
14, 21 and $28 \mathrm{~cm} / \mathrm{s}$ during the first filtration stage. From the result, it can be seen that the modeled pressure drop evolutions overestimated the experimental results for almost all filtration velocities. The 

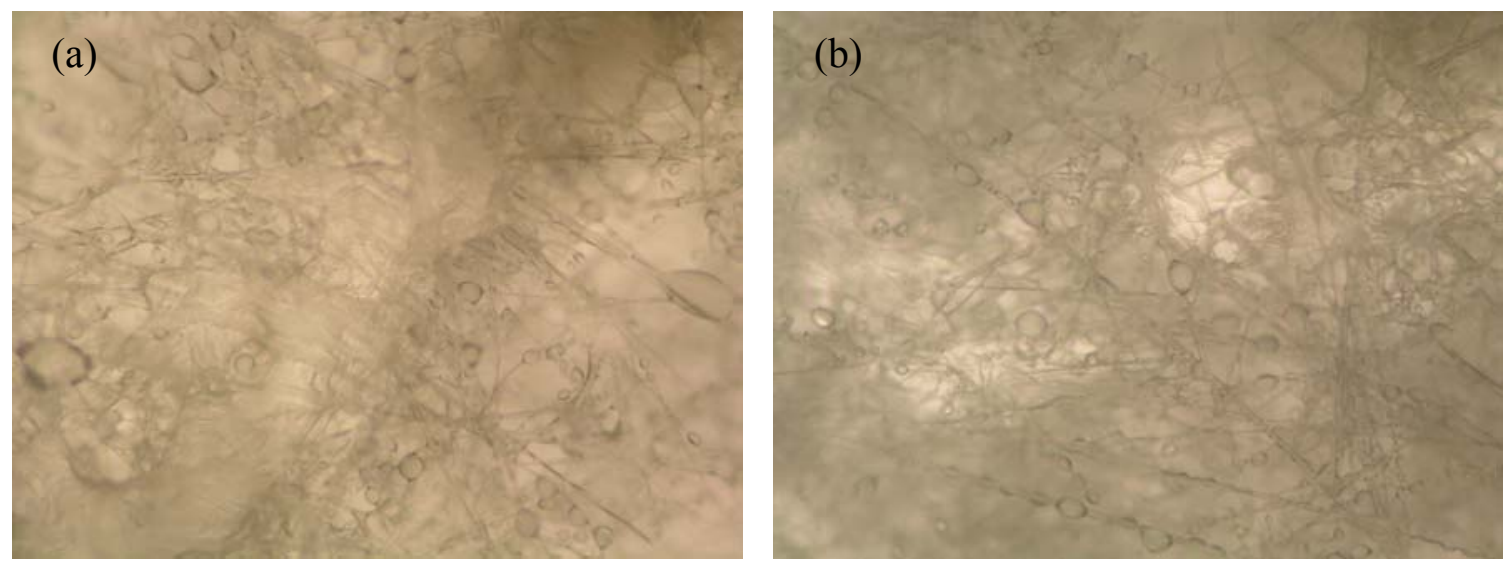

Figs. 10. Optical microscope photographs of a single filter exposed to (a) propylene glycol and (b) palm oil aerosol for $80 \mathrm{~min}(100 \times)$.

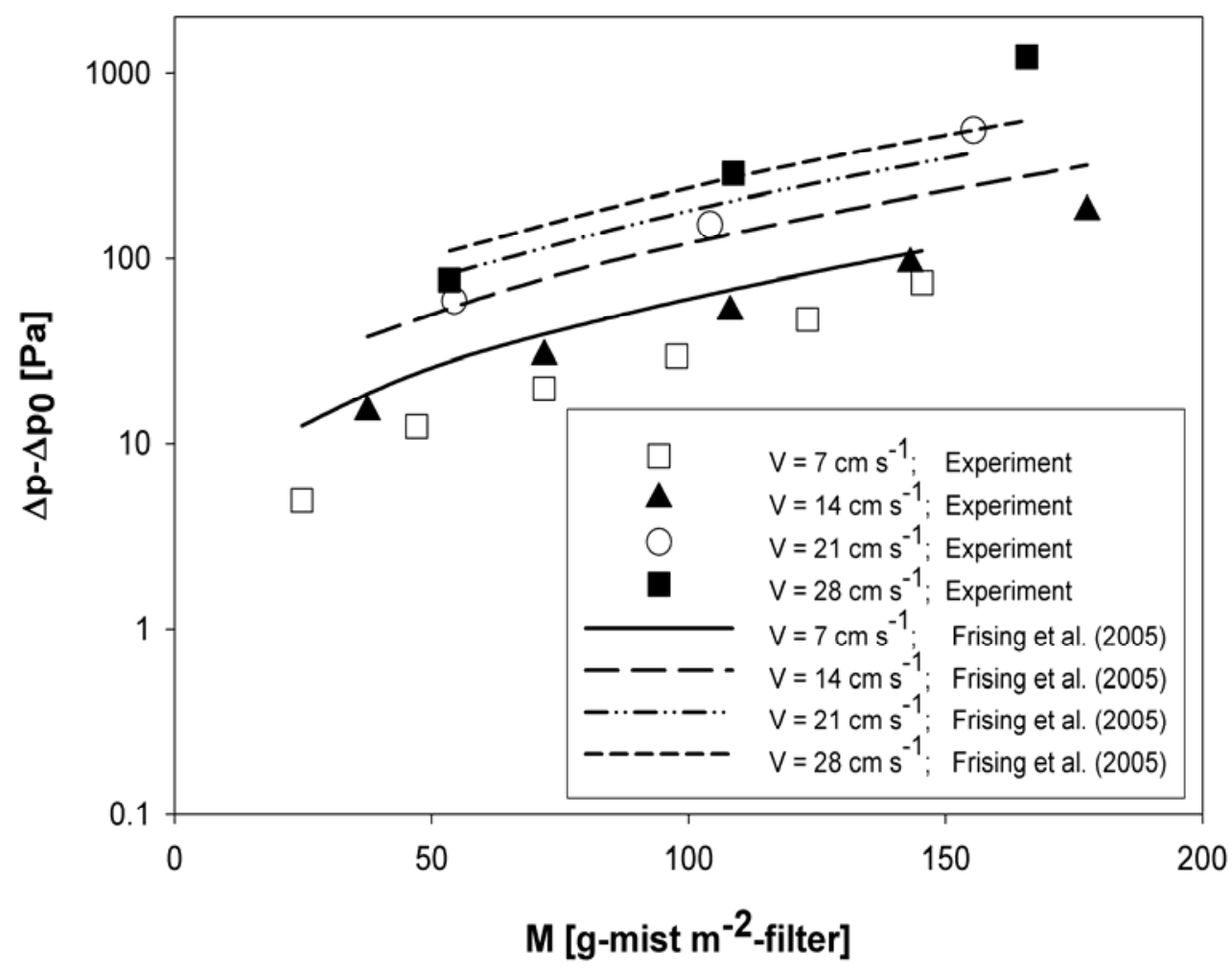

Fig. 11. Comparison between the experimental and modeling values of the pressure drop evolutions of a single filter as a function of collected mass during clogging by propylene glycol aerosol with different filtration velocities during the first filtration stage.

discrepancies are however low, at high velocity. This may be because at the beginning of filtration the deposition of the propylene glycol aerosol particles is made up of droplets deposited around the fibers and form liquid films on the surface of the filter medium but the fibers were not perfectly wetted with liquid while an adapted version of Davies' (1973) 
pressure drop model gives, however, quite satisfactory results for the first filtration stage (Frising et al., 2005) when the liquid wets the fibers perfectly and is uniformly distributed throughout the fibrous filter.

The saturation behavior of the filter when using propylene glycol and palm oil is shown in Figs. 12 and 13, respectively. Air at initial velocities of 7, 14, 21 and $28 \mathrm{~cm} / \mathrm{s}$ was passed through the soaked filters until their weights were constant. This represents the saturation state of the filter at each velocity. Then the velocity was gradually reduced. It was found that the saturation pressure drop from the initial velocity of $7 \mathrm{~cm} / \mathrm{s}$ is greater than that from the initial velocities of 14,21 and 28 $\mathrm{cm} / \mathrm{s}$, respectively for both types of aerosol. The retained mass on the filter sheet saturated with propylene glycol and palm oil, after the pressure drop is constant, decreases when the velocity is increased as shown in Fig. 14. This is because at the higher initial velocity, reentrainment of liquid hold-up is greater. The mass that remains on the filter sheet saturated with palm oil, after the pressure drop is constant, is higher than that saturated with propylene glycol. This result possibly occurs as a result of the viscosity effect. Palm oil is more viscous than propylene glycol so that palm oil cannot re-entrain easily.

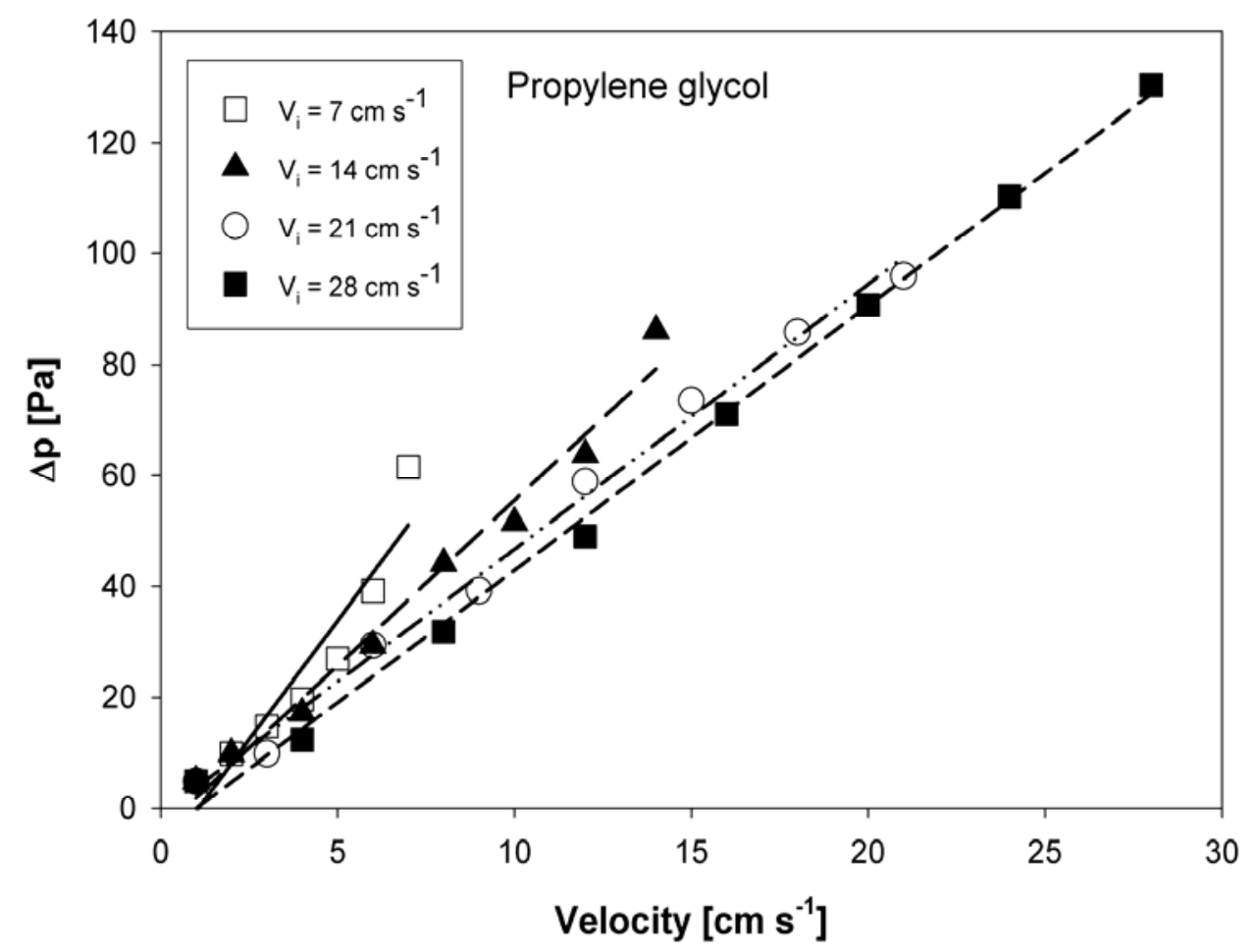

Fig. 12. Saturation pressure drop across the filter sheet saturated with propylene glycol as a function of velocity. 


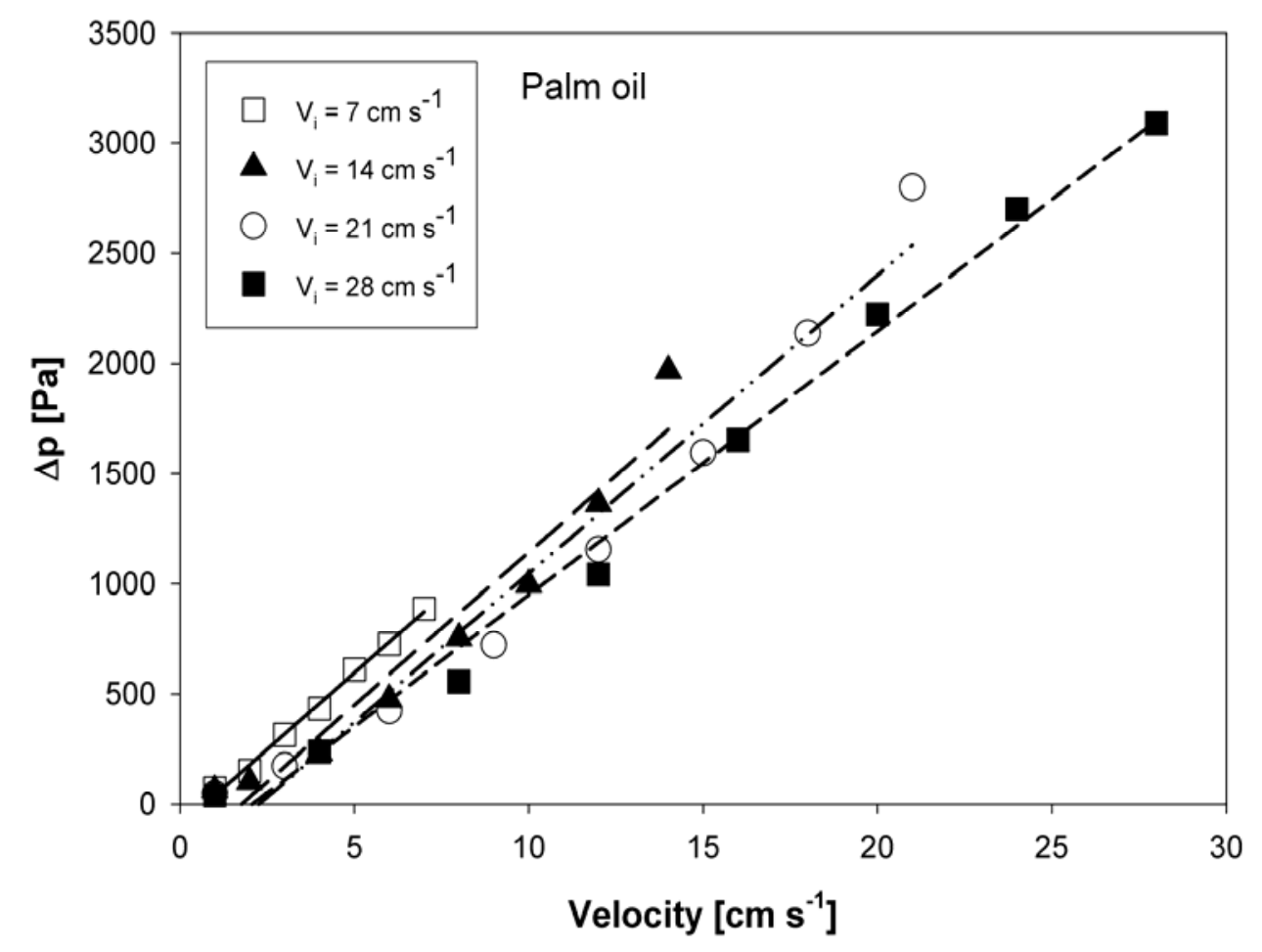

Fig. 13. Saturation pressure drop across the filter sheet saturated with palm oil as a function of velocity.

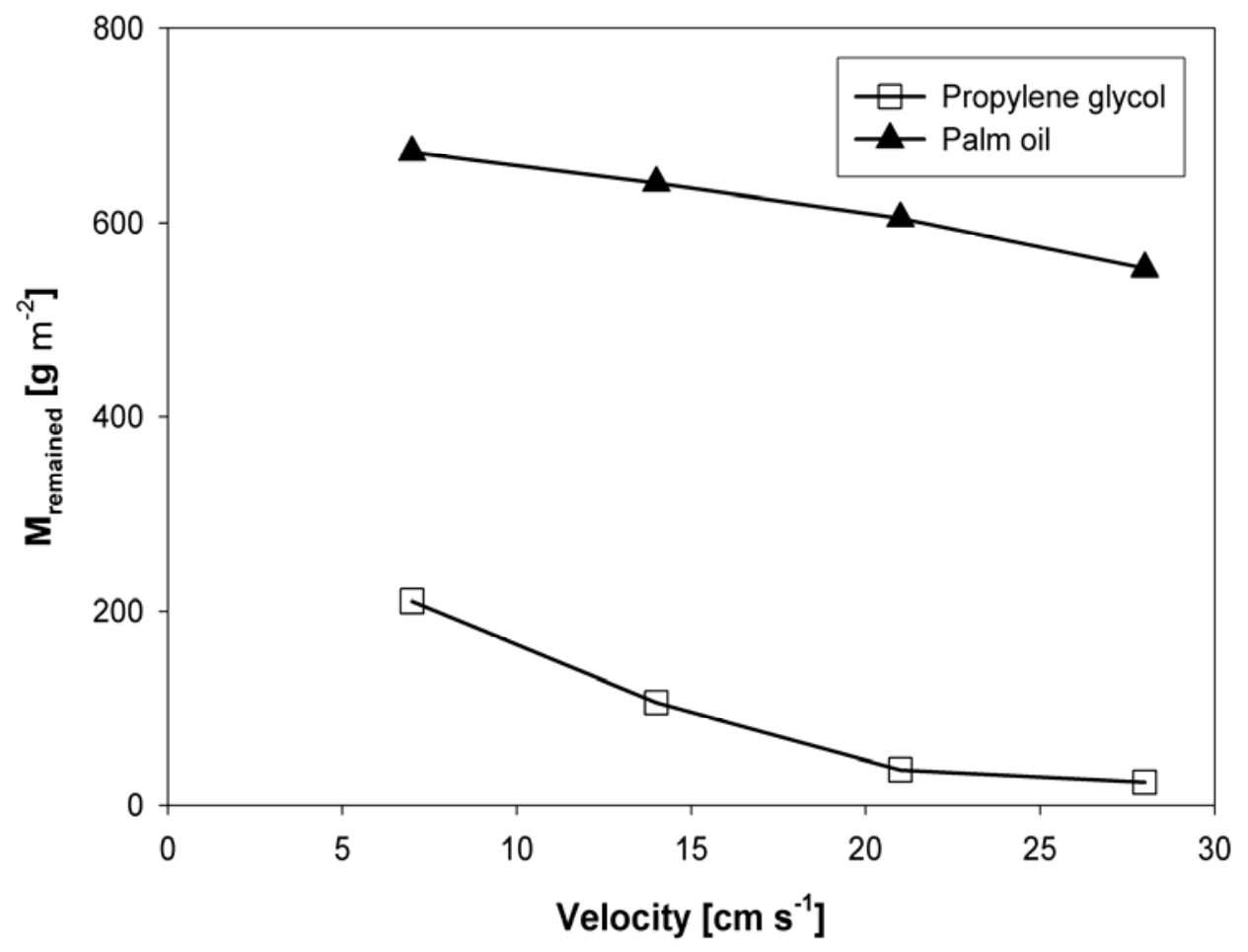

Fig. 14. Retained mass on the filter sheet saturated with propylene glycol and palm oil after the pressure drop is constant as a function of velocity. 


\section{CONCLUSIONS}

The average concentration of propylene glycol is higher than that of palm oil for identical operating condition because propylene glycol is less viscous than palm oil and, hence, easier to be atomized by the Laskin nozzle.

At a low velocity, the efficiency of a virgin filter is very high for all particle sizes due to the influence of the Brownian motion of smaller particles. However, since the particle size range is $0.3-1.0$ micron, Brownian motion should have high influence for the whole range of the particles. That's why the collection efficiency is high at low velocity for all particle sizes. At the highest velocity, the collection efficiency of larger particles is higher for virgin filters than that of small particles due to the influence of the impaction mechanism. The change in pressure drop during clogging increases when the filtration velocity and the number of filter sheets increase. For five filters, while liquid aerosol particles were collected on the surface of the filter, these particles can partially penetrate through the thickness of the medium.

In an initial period, the clogging of the filter by palm oil aerosol is higher than that by propylene glycol aerosol due to the particle size effect. After some period the fibrous filter that is exposed to propylene glycol aerosol has a higher pressure drop because the large propylene glycol droplets deposited on the fibrous filter join to form bridges and liquid films on the surface of the filter.
The saturation pressure drop from the initial velocity of $7 \mathrm{~cm} / \mathrm{s}$ is greater than that from the initial velocities of 14,21 and $28 \mathrm{~cm} / \mathrm{s}$, respectively for both types of liquid. The retained mass on the filter sheet saturated with propylene glycol and palm oil after the pressure drop is constant, is less when the velocity is increased. This is because at the higher initial velocity, re-entrainment of liquid hold-up is greater.

\section{ACKNOWLEDGEMENTS}

Financial support from the Center of Excellence for Innovation in Chemistry (PERCH-CIC), Commission on Higher Education, Ministry of Education is gratefully acknowledged.

\section{REFERENCES}

Contal, P., Simao, J., Thomas, D., Frising, T., Callé, S., Apperet-Collin, J.C. and Bémer, D. (2004). Clogging of Fibre Filters by Submicron Droplets. Phenomena and Influence of Operating Conditions. $J$. Aerosol Sci. 35: 263-278.

Davies, C.N. (1952). The Separation of Airborne Dust and Mist Particles. Proc. Inst. Mech. Engr. 1B: 185-198.

Davies, C.N. (1973). Air Filtration. Academic Press, London.

Frising, T., Thomas, D., Bémer, D. and Contal, P. (2005). Clogging of Fibrous Filters by Liquid Aerosol Particles: Experimental and Phenomenological 
Modeling Study. Chem. Engr. Sci. 60: 2751-2762.

Hinds, W.C. (1999). Aerosol Technol. $2^{\text {nd }}$ ed. John Wiley \& Sons, Inc., New York.

Letts, G.M., Raynor, P.C. and Schumann, R.L. (2003). Selecting Fiber Materials to Improve Mist Filters. J. Aerosol Sci. 34: 1481-1492.

Penicot, P., Thomas, D., Contal, P., Leclerc, D and Vendel, J. (1999). Clogging of HEPA Fibrous Filters by Solid and Liquid Aerosol
Particles: An Experimental Study. Filtr. \& Sep. 36: 59-64

Walsh, D.C., Stenhouse, J.I.T., Scurrah, K.L. and Graef, A. (1996). The Effect of Solid and Liquid Aerosol Particle Loading on Fibrous Filter Material Performance. $J$. Aerosol Sci. 27, Suppl. 1: s617-s618.

Received for review, June 29, 2008 Accepted, July 26, 2008 